\title{
Evolution of endemism on a young tropical mountain
}

Vincent S. F. T. Merckx ${ }^{1,2}$, Kasper P. Hendriks ${ }^{1,3}$, Kevin K. Beentjes ${ }^{1}$, Constantijn B. Mennes ${ }^{1}$, Leontine E. Becking ${ }^{1,4,5}$, Katja T. C. A. Peijnenburg ${ }^{1,6}$, Aqilah Afendy ${ }^{7}$, Nivaarani Arumugam ${ }^{7,8}$, Hugo de Boer ${ }^{1,9,10}$, Alim Biun ${ }^{11}$, Matsain M. Buang ${ }^{11}$, Ping-Ping Chen ${ }^{1}$, Arthur Y. C. Chung ${ }^{12}$, Rory Dow ${ }^{1}$, Frida A. A. Feijen ${ }^{1}$, Hans Feijen ${ }^{1}$, Cobi Feijen-van Soest ${ }^{1}$, József Geml ${ }^{1,2}$, René Geurts $^{13}$, Barbara Gravendeel ${ }^{1,2,14}$, Peter Hovenkamp ${ }^{1}$, Paul Imbun ${ }^{11}$, Isa Ipor ${ }^{15}$, Steven B. Janssens ${ }^{16}$, Merlijn Jocqué ${ }^{17,18}$, Heike Kappes $^{1,19}$, Eyen Khoo ${ }^{12}$, Peter Koomen ${ }^{20}$, Frederic Lens ${ }^{1,2}$, Richard J. Majapun ${ }^{12}$, Luis N. Morgado ${ }^{1}$, Suman Neupane ${ }^{21}$, Nico Nieser ${ }^{1}$, Joan T. Pereira ${ }^{12}$, Homathevi Rahman ${ }^{7}$, Suzana Sabran ${ }^{12}$, Anati Sawang ${ }^{7}$, Rachel M. Schwallier ${ }^{1}$, Phyau-Soon Shim ${ }^{11}{ }_{+}$, Harry Smit ${ }^{1}$, Nicolien Sol ${ }^{1}$, Maipul Spait ${ }^{11}$, Michael Stech ${ }^{1}$, Frank Stokvis ${ }^{1}$, John B. Sugau ${ }^{12}$, Monica Suleiman ${ }^{7}$, Sukaibin Sumail ${ }^{11}$, Daniel C. Thomas ${ }^{1,22,23}$, Jan van Tol ${ }^{1}$, Fred Y. Y. Tuh ${ }^{11}$, Bakhtiar E. Yahya ${ }^{7}$, Jamili Nais ${ }^{11}$, Rimi Repin ${ }^{11}$, Maklarin Lakim ${ }^{11}$ \& Menno Schilthuizen ${ }^{1,2,7}$

Tropical mountains are hot spots of biodiversity and endemism ${ }^{1-3}$, but the evolutionary origins of their unique biotas are poorly understood $^{4}$. In varying degrees, local and regional extinction, long-distance colonization, and local recruitment may all contribute to the exceptional character of these communities ${ }^{5}$. Also, it is debated whether mountain endemics mostly originate from local lowland taxa, or from lineages that reach the mountain by longrange dispersal from cool localities elsewhere ${ }^{6}$. Here we investigate the evolutionary routes to endemism by sampling an entire tropical mountain biota on the 4,095-metre-high Mount Kinabalu in Sabah, East Malaysia. We discover that most of its unique biodiversity is younger than the mountain itself ( 6 million years), and comprises a mix of immigrant pre-adapted lineages and descendants from local lowland ancestors, although substantial shifts from lower to higher vegetation zones in this latter group were rare. These insights could improve forecasts of the likelihood of extinction and 'evolutionary rescue ${ }^{77}$ in montane biodiversity hot spots under climate change scenarios.

In mountainous areas of the humid tropics, steep environmental gradients coincide with high primary productivity and relative climatic stability to sustain large numbers of species, often with striking degrees of endemism at higher elevation $s^{1,8}$. It has therefore been recognized that tropical mountains are biodiversity hot spots of great conservation value ${ }^{1}$, especially because endemics on mountain tops are vulnerable to becoming trapped and then annihilated as a result of global warming ${ }^{2,3}$.

The evolutionary origins of these unique biotas, however, are poorly understood $^{4}$. Like other insular habitats ${ }^{9}$, the endemic biota of an isolated mountain results from complex dynamics among colonization, in situ speciation, and local extinction. Each of these factors is dependent on the age and size of the habitat, and on the environmental contrast between the insular habitat and its matrix ${ }^{10}$. In the case of a tropical mountain top, an added complication is the fact that climate fluctuations may have widened and restricted the geographic range over which the montane conditions have extended in the past, meaning that parts of the endemic biota may be relicts, and other components may be novel in character ${ }^{4,5}$.

Disentangling these possibilities for a single tropical montane biodiversity hot spot requires molecular phylogenetic study of a large number of fauna and flora elements. However, with only few exceptions $s^{11,12}$, evolutionary studies in such hot spots have been limited to single taxa $\mathrm{x}^{13,14}$. This precludes broad understanding of the evolutionary and biogeographic origins of an endemic biota as a whole ${ }^{4}$.

We investigated the evolutionary routes to endemism by sampling an entire tropical mountain biota on the UNESCO World Heritage site of Gunung Kinabalu in Sabah, East Malaysia. We demonstrate that most of its unique biodiversity is younger than the mountain itself and comprises a mix of immigrant pre-adapted lineages as well as descendants from local lowland ancestors.

At 4,095 m, Kinabalu is the tallest mountain between the Himalayas and New Guinea. It is a solitary 'sky island', having emerged during the Pliocene and early Pleistocene as a granite pluton within the surrounding sandstone of the Crocker Range, the latter having formed much earlier, between the Eocene and the early Miocene ${ }^{15}$. Because of the area's tectonic activity, as well as Pleistocene sea level changes, the exact historical progression of its elevation above sea level is not known, but it is likely that a major rise, even beyond today's elevation, of Kinabalu, as well as the central spine of the Crocker Range, took place between 6 million years ago and today (for more geological background see Methods). Since the early days of its exploration ${ }^{16}$, Kinabalu has been famous for its extremely high biological diversity, especially its richness in endemic species, with endemism proportions reaching $25-30 \%$ for some taxa ${ }^{17}$.

To unravel the origins of the exceptionally rich Kinabalu biota, we mounted a Malaysian-Dutch expedition in which 47 taxonomists worked at 37 localities, spanning the full range of elevations (Fig. 1). We used Sanger sequencing to sequence one or more fast-evolving loci for 1,852 individuals, belonging to 18 genera representing gastropods, annelids, insects, arachnids, vertebrates, pteridophytes, bryophytes, and angiosperms. We also obtained 3.7 million basidiomycete

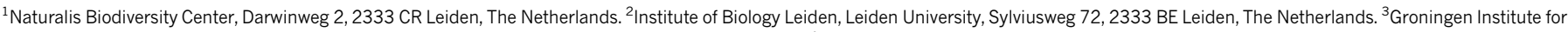

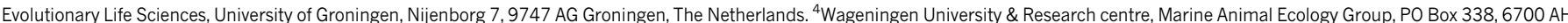

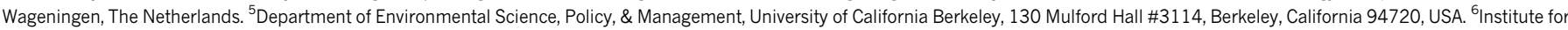

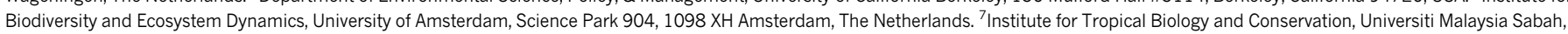

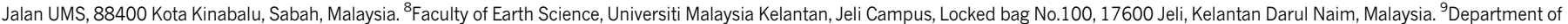

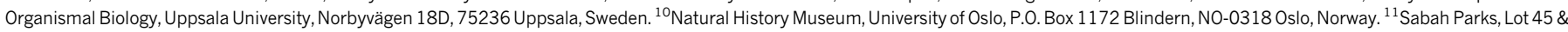

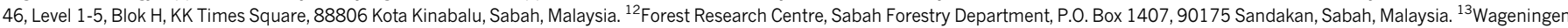
University, Department of Plant Sciences, Laboratory of Molecular Biology, 6700AP Wageningen, The Netherlands. ${ }^{14}$ University of Applied Sciences Leiden, Zernikedreef 11 , 2333 CK Leiden,

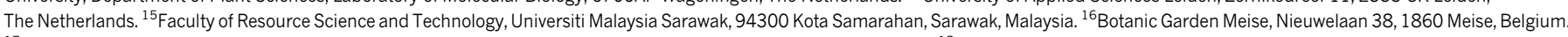

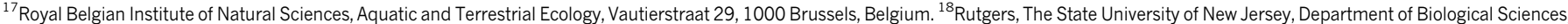
195 University Avenue, Boyden Hall, Newark, New Jersey 07102, USA. ${ }^{19}$ Zoological Institute, University of Cologne, Zülpicher Strasse 47 b, D-50674 Cologne, Germany. ${ }^{20}$ Natuurmuseum Fryslân,

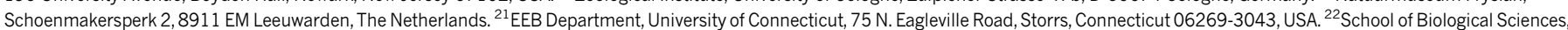
University of Hong Kong, Pok Fu Lam Road, Hong Kong, China. ${ }^{23}$ Singapore Botanic Gardens, 1 Cluny Road, 259569 Singapore, Republic of Singapore.

tDeceased.
} 